PROCEEDINGS OF THE AMERICAN MATHEMATICAL SOCIETY

Volume 127, Number 4, April 1999, Pages 1171-1181

S 0002-9939(99)05053-4

\title{
ESSENTIALLY SUBNORMAL OPERATORS
}

\author{
NATHAN S. FELDMAN
}

(Communicated by David R. Larson)

\begin{abstract}
An operator is essentially subnormal if its image in the Calkin algebra is subnormal. We shall characterize the essentially subnormal operators as those operators with an essentially normal extension. In fact, it is shown that an essentially subnormal operator has an extension of the form "normal plus compact".

The essential normal spectrum is defined and is used to characterize the essential isometries. It is shown that every essentially subnormal operator may be decomposed as the direct sum of a subnormal operator and some irreducible essentially subnormal operators. An essential version of Putnam's Inequality is proven for these operators. Also, it is shown that essential normality is a similarity invariant within the class of essentially subnormal operators. The class of essentially hyponormal operators is also briefly discussed and several examples of essentially subnormal operators are given.
\end{abstract}

\section{INTRODUCTION}

Let $\mathcal{H}$ denote a separable Hilbert space, let $\mathcal{B}(\mathcal{H})$ denote the algebra of all bounded linear operators on $\mathcal{H}$ and let $\mathcal{B}_{0}(\mathcal{H})$ denote the ideal of compact operators. Also, let $\mathcal{B} / \mathcal{B}_{0}$ denote the Calkin algebra and let $\pi: \mathcal{B}(\mathcal{H}) \rightarrow \mathcal{B} / \mathcal{B}_{0}$ denote the natural quotient map.

It is well known that subnormality is a $C^{*}$-property. That is, one may define, in terms of positivity conditions, what it means for any element of a $C^{*}$-algebra to be subnormal. Furthermore, subnormal elements of $C^{*}$-algebras are preserved under *-homomorphisms. This was first shown by Bram [4] in 1955. Also, see Bunce and Deddens [6] or Conway [7], p. 35.

We shall give another proof of the fact that the subnormality is a $C^{*}$-property and even show that subnormality is preserved under certain positive linear maps. Our result shows that the normal spectrum of a subnormal operator is invariant under *-isomorphisms. Thus one may define the normal spectrum of any subnormal element of any $C^{*}$-algebra.

In view of the above remarks, if $\mathcal{A}$ is a $C^{*}$-algebra and $s \in \mathcal{A}$, then it follows that $s$ is subnormal in $\mathcal{A}$ if and only if $\rho(s)$ is a subnormal operator for some (and hence every) faithful representation $\rho$ of $C^{*}(s)$.

Received by the editors August 1, 1997.

1991 Mathematics Subject Classification. Primary 47B20; Secondary 47C15.

Key words and phrases. Essentially subnormal operator, essentially normal operator.

This paper was written while the author was a graduate student working under the direction of Professor John B. Conway. He received partial support from the NSF grant DMS-9401027.

(C)1999 American Mathematical Society 
If $T \in \mathcal{B}(\mathcal{H})$, then we shall say that $T$ is essentially subnormal if $\pi(T)$ is subnormal as an element of $\mathcal{B} / \mathcal{B}_{0}$. More generally, if $P$ is a $C^{*}$-algebra property (such as normal, hyponormal, subnormal, isometry, etc.), then we say that an operator $T$ is essentially $P$ if $\pi(T)$ has property $P$ in $\mathcal{B} / \mathcal{B}_{0}$.

One of the main results of this paper is the following.

Main Result. If $T \in \mathcal{B}(\mathcal{H})$, then the following are equivalent:

(a) $T$ is essentially subnormal;

(b) $T$ has an essentially normal extension;

(c) Thas an extension of the form normal plus compact.

The class of essentially subnormal operators has previously been studied in Lange [11] and Williams [15]. In [11] several conditions are given, each characterizing the essentially subnormal operators whose spectrum is a $K$-spectral set. In [15] it was observed that (b) implies (a) in the above result. He also showed that the converse holds if and only if the Calkin algebra is "dual closed". However, we take a different approach.

If $T$ is essentially subnormal, then, as mentioned above, the normal spectrum of $\pi(T)$ in the Calkin algebra is well defined. We shall call it the essential normal spectrum of $T$. We show that it coincides with the essential spectrum of a "minimal" normal plus compact extension.

We also use the essential normal spectrum to characterize the essential isometries. This leads to some interesting examples and applications such as the diagonalizability of the self-commutator of certain essentially subnormal operators. This is new even for subnormal operators.

For instance, it is shown that if $S$ is the Bergman shift, $\mathcal{M}$ is an invariant subspace for $S$ with infinite index and $T=S \mid \mathcal{M}$, then $T$ is unitarily equivalent to a compact perturbation of the unilateral shift of infinite multiplicity. This answers a question in Conway and Putnam [9] where an irreducible subnormal operator is constructed that is similar to a compact perturbation of the shift of infinite multiplicity. It also implies that the self-commutator of $T,\left[T^{*}, T\right]$, is diagonalizable with eigenvalues of finite multiplicity.

Finally, it is shown that if an essentially subnormal operator $S$ is similar to an essentially normal operator, then $S$ is also essentially normal. This is also new for subnormal operators.

\section{Preliminaries}

If $S$ is a subnormal operator, then let $\sigma_{n}(S)$ denote the normal spectrum of $S$. The following characterization of subnormality is useful when studying $C^{*}$-algebras. Observe that if $S$ is a subnormal operator, then the map from $C\left(\sigma_{n}(S)\right) \rightarrow \mathcal{B}(\mathcal{H})$ given by $f \mapsto S_{f}$, where $S_{f}$ is the Toeplitz operator with symbol $f$, is a positive linear map satisfying $z \mapsto S$ and $\bar{z} z \mapsto S^{*} S$.

Theorem 2.1. An operator $S \in \mathcal{B}(\mathcal{H})$ is subnormal if and only if there exists a compact set $K \subseteq \mathbb{C}$ and a positive linear map $\rho: C(K) \rightarrow \mathcal{B}(\mathcal{H})$ such that $\rho(z)=S$ and $\rho(\bar{z} z)=S^{*} S$. Furthermore, if $K$ and $\rho$ exist, then $\sigma_{n}(S) \subseteq K$.

Remark. All positive linear maps and *-homomorphisms are assumed to be unital (preserve identities). 
Proof. If $\rho$ exists, then since $\rho$ is positive and acts on an abelian $C^{*}$-algebra it must also be completely positive (see [10], p. 260). Thus, we may dilate $\rho$ (see [10], p. 260). That is, there exists a Hilbert space $\mathcal{K} \supseteq \mathcal{H}$ and a ${ }^{*}$-homomorphism $\phi: C(K) \rightarrow \mathcal{B}(\mathcal{K})$ such that $\rho(f)=P \phi(f) \mid \mathcal{H}$ for each $f \in C(K)$, where $P$ denotes the projection of $\mathcal{K}$ onto $\mathcal{H}$. If $N=\phi(z)$, then clearly $N$ is normal and $\sigma(N) \subseteq K$. Since $S=P N \mid \mathcal{H}$ we have that $N=\left[\begin{array}{ll}S & * \\ A & *\end{array}\right]$ relative to $\mathcal{K}=\mathcal{H} \oplus \mathcal{H}^{\perp}$. As $S^{*} S=$ $\rho(\bar{z} z)=P N^{*} N \mid \mathcal{H}$ we must have the $(1,1)$ entry of $N^{*} N$ equal to $S^{*} S$. However,

$$
N^{*} N=\left[\begin{array}{cc}
S^{*} & A^{*} \\
* & *
\end{array}\right]\left[\begin{array}{cc}
S & * \\
A & *
\end{array}\right]=\left[\begin{array}{cc}
S^{*} S+A^{*} A & * \\
* & *
\end{array}\right] .
$$

Thus, $\mathrm{A}=0$. Hence $\mathcal{H}$ is invariant for $N$ and thus $S$ is subnormal. Since $N$ is a normal extension of $S, \sigma_{n}(S) \subseteq \sigma(N) \subseteq K$. Hence the Theorem follows.

Remark. First observe that if $\rho$ and $K$ exist in Theorem 2.1, then $K=\sigma_{n}(S)$ if and only if $\rho$ is one-to-one. Also notice that if $T$ is any operator on $\mathcal{H}$, then there exists a compact set $K \subseteq \mathbb{C}$ and a positive linear map $\rho: C(K) \rightarrow \mathcal{B}(\mathcal{H})$ such that $\rho(z)=T$. This follows because every operator has a normal dilation. In fact, every contraction has a unitary dilation.

Corollary 2.2. If $S$ is a subnormal operator and $\rho: C^{*}(S) \rightarrow \mathcal{B}(\mathcal{H})$ is a positive linear map with $\rho(S)=T$ and $\rho\left(S^{*} S\right)=T^{*} T$, then $T$ is a subnormal operator and $\sigma_{n}(T) \subseteq \sigma_{n}(S)$.

It follows from Corollary 2.2 that the normal spectrum of a subnormal operator is preserved under *-isomorphisms. Thus, if $s$ is a subnormal element of a $C^{*}$-algebra $\mathcal{A}$, then we may define the normal spectrum of $s$. Simply define $\sigma_{n}(s)=\sigma_{n}(\phi(s))$ where $\phi$ is a faithful representation of $C^{*}(s)$. By Corollary 2.2 this is independent of the choice of $\phi$.

Corollary 2.3. If $\mathcal{A}$ is a $C^{*}$-algebra and $s \in \mathcal{A}$, then $s$ is subnormal in $\mathcal{A}$ if and only if there exists a compact set $K \subseteq \mathbb{C}$ and a positive linear map $\rho: C(K) \rightarrow \mathcal{A}$ such that $\rho(z)=s$ and $\rho(\bar{z} z)=s^{*} s$. Furthermore, if $\rho$ exists, then $\sigma_{n}(s) \subseteq K$.

Observe that if $s \in \mathcal{A}$ is subnormal, then we may choose $K=\sigma_{n}(s)$.

We now prove a general decomposition theorem for operators that will be useful later on. Say that an operator is completely reducible if its lattice of reducing subspaces has no non-zero minimal elements. Thus a normal operator is completely reducible if and only if it has no eigenvalues.

Proposition 2.4. If $T$ is an operator, then $T=S_{0} \oplus\left(\bigoplus_{n=1}^{\infty} S_{n}\right) \oplus\left(\bigoplus_{n=1}^{\infty} T_{n}\right)$ where

(a) $S_{0}$ is completely reducible;

(b) $S_{n}$ is irreducible and $C^{*}\left(S_{n}\right) \cap \mathcal{B}_{0}=(0)$;

(c) $T_{n}$ is irreducible and $\mathcal{B}_{0} \subseteq C^{*}\left(T_{n}\right)$.

Naturally, not every summand need appear in the above decomposition.

Lemma 2.5. If $T$ is an operator and $C^{*}(T)$ contains a non-zero compact operator, then $T$ has a non-zero irreducible orthogonal summand $S$ such that $\mathcal{B}_{0} \subseteq C^{*}(S)$.

Proof. If $\mathcal{I}=C^{*}(T) \cap \mathcal{B}_{0}$, then $\mathcal{I}$ is a non-zero $C^{*}$-algebra of compact operators. However, every $C^{*}$-algebra of compact operators is unitarily equivalent to a direct sum of algebras of the form $\mathcal{B}_{0}(\mathcal{M})$ where $\mathcal{M}$ is a reducing subspace for $\mathcal{I}$ (see 
[10], p. 39). Thus, there is a reducing subspace $\mathcal{M}$ for $\mathcal{I}$ such that $\mathcal{I} \mid \mathcal{M}=\mathcal{B}_{0}(\mathcal{M})$. Since $\mathcal{I}$ is an ideal in $C^{*}(T)$ that acts non-degenerately on $\mathcal{M}$ it follows that $\mathcal{M}$ is reducing for $T$. If $S=T \mid \mathcal{M}$, then $S$ is the required orthogonal summand.

Remark. It follows from Lemma 2.5 that if $T$ is completely reducible, then $C^{*}(T)$ contains no non-zero compact operators.

Proof of Proposition 2.4. If $T$ is irreducible, then by Lemma $2.5 T$ is either of the form (b) or (c). Likewise, if $T$ has no minimal reducing subspaces, then $T$ is completely reducible so it has the form in (a). Otherwise, $T$ is reducible and has some minimal reducing subspaces. If $T$ acts on $\mathcal{H}$, then let $\left\{\mathcal{H}_{n}: n \geq 1\right\}$ be a maximal collection of pairwise orthogonal minimal reducing subspaces for $T$. This gives that $T=S_{0} \oplus\left(\bigoplus_{n=1}^{\infty} B_{n}\right)$ where $\mathcal{H}=\mathcal{H}_{0} \oplus \bigoplus_{n=1}^{\infty} \mathcal{H}_{n}, B_{n}=T \mid \mathcal{H}_{n}$ for $n \geq 1$ and $S_{0}=T \mid \mathcal{H}_{0}$. Clearly $B_{n}$ is irreducible since each $\mathcal{H}_{n}$ is minimal. Likewise, $S_{0}$ is completely reducible by the maximality of the collection $\left\{\mathcal{H}_{n}\right\}$. Thus, by Lemma 2.5 each $B_{n}$ has the form of (b) or (c). So the result follows.

\section{MAin Results}

Theorem 3.1. If $T \in \mathcal{B}(\mathcal{H})$, then the following are equivalent:

(a) $T$ is essentially subnormal;

(b) Thas an essentially normal extension;

(c) Thas an extension of the form normal plus compact.

Proof. It is clear that (c) implies (b). Let us show that (b) implies (a). There are several ways to do this depending on your favorite definition of subnormality. For completeness we shall use Corollary 2.3. If $R \in \mathcal{B}(\mathcal{K})$ is an essentially normal extension of $T$, then the compression map $\kappa: \mathcal{B}(\mathcal{K}) \rightarrow \mathcal{B}(\mathcal{H})$ is a positive linear map that sends compact operators to compact operators. Hence $\kappa$ induces a positive linear map $\hat{\kappa}: \mathcal{B}(\mathcal{K}) / \mathcal{B}_{0}(\mathcal{K}) \rightarrow \mathcal{B}(\mathcal{H}) / \mathcal{B}_{0}(\mathcal{H})$. Furthermore, it is easy to see that $\hat{\kappa}(\pi(R))=\pi(T)$ and $\hat{\kappa}\left(\pi\left(R^{*} R\right)\right)=\pi\left(T^{*} T\right)$. Thus, since $\pi(R)$ is normal in $\mathcal{B}(\mathcal{K}) / \mathcal{B}_{0}(\mathcal{K})$ it follows from Corollary 2.3 that $\pi(T)$ is subnormal in $\mathcal{B}(\mathcal{H}) / \mathcal{B}_{0}(\mathcal{H})$. Observe that it also follows from Corollary 2.3 that $\sigma_{n}(\pi(S)) \subseteq \sigma_{n}(\pi(R))=\sigma_{e}(R)$.

We now show that (a) implies (c). If $T$ is essentially subnormal, then $\pi(T)$ is subnormal in $\mathcal{B}(\mathcal{H}) / \mathcal{B}_{0}(\mathcal{H})$. Thus by Corollary 2.3 there exists a compact set $K$ (that we may choose to be $\sigma_{n}(\pi(T))$ ) and a positive linear map $\rho: C(K) \rightarrow$ $\mathcal{B}(\mathcal{H}) / \mathcal{B}_{0}(\mathcal{H})$ such that $\rho(z)=T$ and $\rho(\bar{z} z)=T^{*} T$. It follows from [10], p. 266, that there exists a positive (unital) linear map $\tau: C(K) \rightarrow \mathcal{B}(\mathcal{H})$ such that $\rho=\pi \circ \tau$. Since $\tau$ is positive, it is also completely positive (see [10], p. 260). Thus, $\tau$ has a dilation (see [10], p. 260). That is, there exists a Hilbert space $\mathcal{K} \supseteq \mathcal{H}$ and a *- $^{-}$ homomorphism $\phi: C(K) \rightarrow \mathcal{B}(\mathcal{K})$ such that $\tau(f)=P \phi(f) \mid \mathcal{H}$ for each $f \in C(K)$; where $P$ denotes the projection of $\mathcal{K}$ onto $\mathcal{H}$.

If $N=\phi(z)$, then $N$ is normal and $\sigma(N) \subseteq K$. Also let $S=\tau(z)$. Since $S=P N \mid \mathcal{H}$ we have that $N=\left[\begin{array}{ll}S & * \\ A & *\end{array}\right]$ relative to $\mathcal{K}=\mathcal{H} \oplus \mathcal{H}^{\perp}$. As $\pi\left(S^{*} S\right)=$ $\pi\left(T^{*} T\right)=\rho(\bar{z} z)=\pi(\tau(\bar{z} z))=\pi\left(P N^{*} N \mid \mathcal{H}\right)$, we must have the $(1,1)$ entry of $N^{*} N$ equal to a compact perturbation of $S^{*} S$. However,

$$
N^{*} N=\left[\begin{array}{cc}
S^{*} & A^{*} \\
* & *
\end{array}\right]\left[\begin{array}{ll}
S & * \\
A & *
\end{array}\right]=\left[\begin{array}{cc}
S^{*} S+A^{*} A & * \\
* & *
\end{array}\right] .
$$


Thus, $A$ is compact. Since $S$ is a compact perturbation of $T,(T-S)$ is compact. So, if $C=\left[\begin{array}{cc}(T-S) & 0 \\ -A & 0\end{array}\right]$, then $C$ is compact and $N+C=\left[\begin{array}{ll}T & * \\ 0 & *\end{array}\right]$ is an extension of $T$. So, the result follows.

Remark. It follows from the proof of (b) implies (a) that the restriction of an essentially subnormal operator to an invariant subspace is essentially subnormal.

Corollary 3.2. Let $T$ be an essentially subnormal operator.

(a) If $R$ is an essentially normal extension of $T$, then $\sigma_{n}(\pi(T)) \subseteq \sigma_{e}(R)$.

(b) There exists an extension $R$ of $T$ of the form normal plus compact, such that $\sigma_{e}(R)=\sigma_{n}(\pi(T))$.

Remark. Recall that $\sigma_{n}(\pi(T))$ is defined as the normal spectrum of $\phi(\pi(T))$ for some faithful representation $\phi$ of $C^{*}(\pi(T))$. The above corollary relates this to extensions of $T$.

Proof. (a) It follows from the above proof of (b) implies (a) in Theorem 3.1 that if $R$ is an essentially normal extension of $T$, then $\sigma_{n}(\pi(T)) \subseteq \sigma_{e}(R)$.

(b) This also follows from the proof of (a) implies (c) in Theorem 3.1 where we may choose the compact set $K=\sigma_{n}(\pi(T)$ ) (as mentioned in the proof). This implies the existence of an extension of the form normal plus compact with essential spectrum contained in $\sigma_{n}(\pi(T))$. The reverse inclusion follows from (a).

Proposition 3.3. If $S$ is an essentially subnormal operator, then the following hold.

(a) If $f$ is analytic near $\sigma(S)$, then $\sigma_{n}(\pi(f(S)))=f\left(\sigma_{n}(\pi(S))\right)$.

(b) $\sigma_{l e}(S) \subseteq \sigma_{n}(\pi(S)) \subseteq \sigma_{e}(S)$.

(c) $\sigma_{e}(S)=\sigma_{n}(\pi(S)) \cup\left\{\right.$ some components of $\left.\mathbb{C}-\sigma_{n}(\pi(S))\right\}$.

(d) If $\mathcal{M} \in$ LatS, then $\sigma_{n}(\pi(S \mid \mathcal{M})) \subseteq \sigma_{n}(\pi(S))$.

(e) If $C^{*}(S) \cap \mathcal{B}_{0}=(0)$, then $S$ is subnormal.

Proof. Let $\phi$ be a faithful representation of $C^{*}(\pi(S))$ on a separable Hilbert space. Also let $T=\phi(\pi(S))$. Thus $T$ is a subnormal operator and, by definition, $\sigma_{n}(\pi(S))$ $=\sigma_{n}(T)$; see the remarks after Corollary 2.2.

Since $\phi$ is a *-isomorphism and $f$ is analytic in a neighborhood of $\sigma(S)$, we have $\sigma_{n}(\pi(f(S)))=\sigma_{n}(f(T))=f\left(\sigma_{n}(T)\right)=f\left(\sigma_{n}(\pi(S))\right)$. Where the second equality follows from Conway [7], p. 42.

Observe that (b) follows from the fact that $\sigma_{l}(T)=\sigma_{a p}(T) \subseteq \sigma_{n}(T) \subseteq \sigma(T)$. Likewise, (c) follows from the corresponding identity for $T$ (see Conway [7], p. 41).

For (d), first use Corollary 3.2 to find an essentially normal extension $R$ of $S$ with $\sigma_{e}(R)=\sigma_{n}(\pi(S))$. Now, since $R$ is also an extension of $S \mid \mathcal{M}$, Corollary 3.2 also gives that $\sigma_{n}(\pi(S \mid \mathcal{M})) \subseteq \sigma_{e}(R)=\sigma_{n}(\pi(S))$.

(e) If $C^{*}(S) \cap \mathcal{B}_{0}=(0)$, then the quotient map $\pi$ is a *isomorphism of $C^{*}(S)$ onto $C^{*}(\pi(S))$. Since $S$ is essentially subnormal, $\pi(S)$ is subnormal. Thus, since subnormality is a $C^{*}$-property, $S$ is also subnormal.

We now prove an essential version of Putnam's Inequality for essentially subnormal operators as well as a decomposition theorem. If $K$ is a compact set in $\mathbb{C}$, then $R(K)$ denotes the uniform closure of the set of rational functions with poles off $K$. 
Theorem 3.4. If $T$ is an essentially subnormal operator, then

(a) $\left\|\left[T^{*}, T\right]\right\|_{e} \leq \operatorname{dist}\left[\bar{z}, R\left(\sigma_{e}(T)\right)\right]^{2}$;

(b) $T=S \oplus\left(\bigoplus_{n=1}^{\infty} T_{n}\right)$ where $S$ is a subnormal operator and $T_{n}$ is an irreducible essentially subnormal operator with $\mathcal{B}_{0} \subseteq C^{*}\left(T_{n}\right)$.

Remark. Part (a) was first proven for subnormal operators by Aleman [1] using different methods. The proof given here first appeared in Conway and Feldman [8] for subnormal operators.

Proof. (a) Let $\phi$ be a faithful representation of $C^{*}(\pi(T))$ and set $S=\phi(\pi(T))$. Since $T$ is essentially subnormal, $S$ is subnormal. Since $\phi$ is an isometry we have

$$
\left\|\left[T^{*}, T\right]\right\|_{e}=\left\|\left[S^{*}, S\right]\right\| \leq \operatorname{dist}[\bar{z}, R(\sigma(S))]^{2}=\operatorname{dist}\left[\bar{z}, R\left(\sigma_{e}(T)\right)\right]^{2} .
$$

Where we have used a version of Putnam's Inequality for subnormal operators due to Axler and Shapiro [3] (also see Conway [7], p. 177).

(b) By Proposition 2.4, we know that $T=S_{0} \oplus\left(\bigoplus_{n=1}^{\infty} S_{n}\right) \oplus\left(\bigoplus_{n=1}^{\infty} T_{n}\right)$ where $S_{0}$ is completely reducible, $C^{*}\left(S_{n}\right)$ contains no non-zero compact operators and $\mathcal{B}_{0} \subseteq C^{*}\left(T_{n}\right)$. If we let $S=S_{0} \oplus\left(\bigoplus_{n=1}^{\infty} S_{n}\right)$, then $S$ is essentially subnormal and $C^{*}(S)$ contains no non-zero compact operators. Thus, by Proposition 3.3(e), $S$ is subnormal. Furthermore, each $T_{n}$ is essentially subnormal, since they each have $S$ as an essentially subnormal extension.

Remark. There is also a version of Theorem 3.4 (a) for essentially hyponormal operators. That is, if $T$ is essentially hyponormal, then $\left\|\left[T^{*}, T\right]\right\|_{e} \leq \frac{1}{\pi} \operatorname{Area}\left[\sigma_{e}(T)\right]$. This follows from Putnam's Inequality for hyponormal operators and an argument as above.

Example 3.5. (a) $S+C$ is essentially subnormal whenever $S$ is subnormal and $C$ is compact.

(b) $S^{*} \mid \mathcal{M}^{\perp}$ is essentially subnormal whenever $S$ is a subnormal operator with a compact self-commutator and $\mathcal{M} \in$ Lat $S$.

(c) If $S$ is a subnormal operator, then the Toeplitz operator, $S_{f}$, with symbol $f \in L^{\infty}(\mu)$ is essentially subnormal whenever the Hankel operator $H_{f}$ is compact.

(d) $S^{(\infty)}$ is essentially subnormal if and only if $S$ is subnormal.

(e) $S$ and $S^{*}$ are essentially subnormal if and only if $S$ is essentially normal.

Proof. Parts (a) and (b) are clear. (d) follows from Proposition 3.3(e). Consider (c). If $S$ is a subnormal operator, $N$ is the minimal normal extension of $S$ and $\mu=\operatorname{svsm} N$, then for each $f \in L^{\infty}(\mu), f(N)=\left[\begin{array}{ll}S_{f} & * \\ H_{f} & *\end{array}\right]$. Thus, if the Hankel operator $H_{f}$ is compact, then $S_{f}$ has $f(N)+\left[\begin{array}{cc}0 & 0 \\ -H_{f} & 0\end{array}\right]$ as an extension. So, $S_{f}$ is essentially subnormal.

(e) By considering a faithful representation of $C^{*}(\pi(S))$, one easily sees that if $S$ is essentially subnormal, then it is also essentially hyponormal. Thus, if both $S$ and $S^{*}$ are essentially subnormal, then the self-commutator of $\pi(S)$ in $\mathcal{B} / \mathcal{B}_{0}$ is both positive and negative, hence zero. So, $S$ is essentially normal. The converse is clear.

Example 3.6. If $f \in L^{\infty}(\partial \mathbb{D})$, then let $T_{f}$ denote the Toeplitz operator with the symbol $f$ on $H^{2}$. 
(a) If $f, \bar{f} \in H^{\infty}+C(\partial \mathbb{D})$, then $T_{f}$ is essentially normal.

(b) If $f \in H^{\infty}+C(\partial \mathbb{D})$, then $T_{f}$ is essentially subnormal.

(c) If $f=g+\bar{h}$ where $g, h \in H^{\infty}$ and $h$ is in the Dirichlet space, then $T_{f}$ is essentially subnormal.

Example 3.7. There exists an essentially hyponormal unilateral weighted shift that is not essentially normal.

Proof. Define two sequences $\left\{\alpha_{n}\right\}_{n=0}^{\infty}$ and $\left\{\beta_{n}\right\}_{n=0}^{\infty}$. Let $\alpha_{n}=1$ if $n=2^{k}$ for some $k \geq 2$ and $\alpha_{n}=0$, otherwise. Also, let $\beta_{n}=\frac{-1}{k}$ if $2^{k}+1 \leq n \leq 2^{k}+k$ and $k \geq 2$. Otherwise, $\beta_{n}=0$.

One easily checks that $0 \leq \sum_{j=1}^{n} \alpha_{j}-\sum_{j=1}^{n}\left|\beta_{j}\right| \leq 1$ for all $n \geq 0$. Now define a unilateral weighted shift $T$ with weights $w_{n}^{2}=\sum_{j=1}^{n} \alpha_{j}-\sum_{j=1}^{n}\left|\beta_{j}\right|$.

One easily checks that $\left[T^{*}, T\right]$ has eigenvalues $\left\{\alpha_{j}+\beta_{j}\right\}$. Thus, since $\beta_{j} \rightarrow 0$ and $\alpha_{j} \geq 0,\left[T^{*}, T\right]$ is a compact perturbation of a positive operator. So, $T$ is essentially hyponormal. However, since $\left\{\alpha_{j}+\beta_{j}\right\}$ does not converge to zero, $\left[T^{*}, T\right]$ is not compact.

Finally, if one wants $T$ to have all positive weights, then choose a sequence $\varepsilon_{j}>0$ with $\varepsilon_{j} \rightarrow 0$, and replace any $w_{j}$ that equals zero with an $\varepsilon_{j}$.

Almost surely, there exists an essentially subnormal unilateral weighted shift that is not essentially normal.

Question 3.8. Can one characterize the weighted shifts that are essentially subnormal?

\section{Essential isometries}

Recall that $T$ is an isometry if and only if $T^{*} T=I$. Thus, isometries may be defined in any $C^{*}$-algebra. In particular, we may speak of essential isometries as those operators $T$ such that $\pi(T)$ is an isometry in $\mathcal{B} / \mathcal{B}_{0}$. That is, $T^{*} T-I$ is compact. For example, a unilateral weighted shift with weights $\left\{w_{n}\right\}$ is an essential isometry if and only if $w_{n} \rightarrow 1$ as $n \rightarrow \infty$.

One of the most well understood class of subnormal operators is the class of isometries. Thus one may expect that the class of essential isometries would be a nice collection of essentially subnormal operators in which several natural questions may be answered. We will obtain a complete classification of all essential isometries. An application of this allows us to show that certain subnormal operators have diagonalizable self-commutators. Also, some natural questions are raised about unitary equivalence modulo the compacts for essentially subnormal operators.

Proposition 4.1. If $S$ is an essentially subnormal operator, then the following are equivalent:

(a) $S$ is an essential isometry;

(b) $\sigma_{n}(\pi(S)) \subseteq \partial \mathbb{D}$;

(c) $S$ has an extension of the form unitary plus compact;

(d) $S$ has an essentially normal extension $R$ with $\sigma_{e}(R) \subseteq \partial \mathbb{D}$.

Proof. Let $\phi$ be a faithful representation of $C^{*}(\pi(S))$ and set $T=\phi(\pi(S))$. Thus, $T$ is subnormal. Furthermore, $T$ is an isometry if and only if $S$ is an essential isometry. By definition, $\sigma_{n}(\pi(S))=\sigma_{n}(T)$. Furthermore, its well known that $T$ is an isometry if and only if $\sigma_{n}(T) \subset \partial \mathbb{D}$. Thus (a) and (b) are equivalent. 
If (b) holds, then by Corollary 3.2 there exists an extension of $S$ of the form $N+C$ with $N$ normal and $C$ compact having the property that $\sigma_{e}(N) \subset \sigma_{n}(\pi(S))$. Since $\sigma_{n}(\pi(S)) \subset \partial \mathbb{D}$, a compact perturbation of $N$ is a unitary operator. Say, $N+K=U$ where $K$ is compact and $U$ is unitary. Thus, $U+(C-K)$ is an extension of $S$ with the required form.

Clearly, (c) implies (d). Also, (d) implies (b) by Corollary 3.2. So the result follows.

If $S$ is an essentially subnormal operator, then $\sigma_{l e}(S) \subseteq \sigma_{n}(\pi(S))$. Thus, if $S$ is an essential isometry, then the Fredholm index of $S$, ind $S$, is well-defined.

If $S$ is an essential isometry that is also essentially normal, then $S$ is actually essentially unitary. Furthermore, these have all been classified by the BrownDouglas-Filmore Theory [5]. Thus an essentially unitary operator is a compact perturbation of either a unitary, the unilateral shift of multiplicity $n$ or its adjoint (the backward shift) where the value of $n$ depends on ind $S$.

Thus it remains to consider the essential isometries that are not essentially normal.

Theorem 4.2. If $S$ is essentially subnormal, then the following are equivalent:

(a) $S$ is an essential isometry that is not essentially normal;

(b) $S$ is unitarily equivalent to a compact perturbation of the unilateral shift of infinite multiplicity.

Proof. It suffices to show (a) implies (b). By Proposition $4.1 \sigma_{l e}(S) \subseteq \sigma_{n}(\pi(S)) \subseteq$ $\partial \mathbb{D}$. Thus, $S$ has closed range and a finite dimensional kernel. Hence, by compactly perturbing $S$ we may assume that $S$ is one-to-one. Since $S$ is an essential isometry, $S^{*} S=I+K$ where $I$ is the identity and $K$ is compact. Thus, also $|S|=I+K$ for some new compact operator $K$. By the polar decomposition of $S, S=W|S|=$ $W(I+K)=W+C$ where $W$ is a partial isometry and $C$ is compact. Since $S$ is one-to-one, so is $|S|$ and $W$. In particular, $W$ is an isometry. Consider the Wold decomposition of $W$. Say, $W \cong U^{(n)} \oplus V$ where $U$ is a unilateral shift (of multiplicity one) and $V$ is a unitary. Notice that $n=\infty$ since $S$ is not essentially normal. Since $U \oplus V$ is a compact perturbation of $U$, we have that $W \cong U^{(\infty)} \oplus V \cong$ $U^{(\infty)} \oplus U \oplus V \cong{ }_{\left(\bmod \mathcal{B}_{0}\right)} U^{(\infty)} \oplus U \cong U^{(\infty)}$. Thus, $W$ is a compact perturbation of $U^{\infty}$.

If $S$ is a subnormal operator, then let $\operatorname{svsm} S$ denote the scalar-valued spectral measure of the minimal normal extension of $S$. If $0 \notin \sigma_{a p}(S)$ and $\mathcal{M} \in \operatorname{Lat} S$, then the index of $\mathcal{M}$ is defined to be $-\operatorname{ind}(S \mid \mathcal{M})$.

Corollary 4.3. If $S$ is a pure subnormal operator such that $\sigma_{e}(S)=\partial \mathbb{D}$ and $\mathcal{M} \in$ LatS has infinite index, then $S \mid \mathcal{M}$ is unitarily equivalent modulo the compacts to the unilateral shift of infinite multiplicity.

Proof. It follows from Theorem 3.4(a) that $S$ is essentially normal. Hence, by Proposition 4.1 (d) we see that $S \mid \mathcal{M}$ is an essential isometry. So, Theorem 4.2 applies.

Corollary 4.4. Let $S$ be a pure subnormal operator with $\sigma_{e}(S)=\partial \mathbb{D}$ and svsmS carried by $\mathbb{D}$. If $\mathcal{M} \in$ LatS has infinite index and $T=S \mid \mathcal{M}$, then $\left[T^{*}, T\right]$ is diagonalizable with non-zero eigenvalues having finite multiplicity. Furthermore, $\sigma_{e}\left(\left[T^{*}, T\right]\right)=\{0,1\}$. 
Proof. By Corollary 4.3, $T$ is a compact perturbation of $U^{(\infty)}$. Thus, $\left[T^{*}, T\right]$ is a compact perturbation of an infinite rank projection. Hence, $\sigma_{e}\left(\left[T^{*}, T\right]\right)=\{0,1\}$. Since the $\operatorname{svsm}\left[T^{*}, T\right]$ is purely atomic, $\left[T^{*}, T\right]$ is diagonalizable. Furthermore, since $\operatorname{svsm} S$ is carried by $\mathbb{D}, S$ strictly decreases the norm of each vector. Thus, 1 is not an eigenvalue of $\left[T^{*}, T\right]$. So, every non-zero eigenvalue of $\left[T^{*}, T\right]$ has finite multiplicity, since it does not belong to $\sigma_{e}\left(\left[T^{*}, T\right]\right)$.

Example 4.5. If $B$ is the Bergman shift, $\mathcal{M} \in \operatorname{Lat} B$ has infinite index and $S=B \mid \mathcal{M}$, then $\mathrm{S}$ is an irreducible subnormal operator that is unitarily equivalent modulo the compacts to the unilateral shift of infinite multiplicity.

Thus it follows that $\left[S^{*}, S\right]$ is diagonalizable with eigenvalues of finite multiplicity. Furthermore, the eigenvalues cluster only at zero and one.

The previous example answers a question in Conway and Putnam [9] where an irreducible subnormal operator is constructed that is similar to a compact perturbation of the unilateral shift of infinite multiplicity. They ask whether "similar" can be replaced by "unitary equivalence". The work of Apostol, Bercovici, Foias and Pearcy [2] implies that if $S$ is a pure subnormal operator with $\sigma_{e}(S)=\partial \mathbb{D}$ and $\operatorname{svsm} S$ carried by $\mathbb{D}$, then $S$ has invariant subspaces with infinite index. Also, see Hedenmalm, Richter and Seip [12] for a construction of such invariant subspaces in the Bergman space.

Example 4.5 shows that one may compactly perturb the unilateral shift of infinite multiplicity and obtain an irreducible subnormal operator. Can this be done with the inflation of other operators?

Question 4.6. Is there an irreducible subnormal operator unitarily equivalent mod the compacts to the Bergman shift of infinite multiplicity?

The following simple proposition shows that the unilateral shift of infinite multiplicity and the Bergman shift of infinite multiplicity are not equivalent mod the compacts.

Proposition 4.7. If $S$ and $T$ are operators such that $\mathcal{B}_{0} \subseteq C^{*}(S)$ and $T$ is irreducible, then the following are equivalent:

(a) $S \cong T$;

(b) $S^{(\infty)} \cong T^{(\infty)}$;

(c) $S^{(\infty)} \cong T^{(\infty)}\left(\bmod \mathcal{B}_{0}\right)$.

Proof. It suffices to show that (c) implies (a). If $S^{(\infty)} \cong T^{(\infty)}\left(\bmod \mathcal{B}_{0}\right)$, then $C^{*}(S) \approx C^{*}\left(\pi\left(S^{(\infty)}\right)\right) \approx C^{*}\left(\pi\left(T^{(\infty)}\right)\right) \approx C^{*}(T)$. Where $C^{*}(a) \approx C^{*}(b)$ means that the map $a \mapsto b$ extends to a *-isomorphism. Thus, $C^{*}(S) \approx C^{*}(T)$. Let $\phi$ denote this ${ }^{*}$-isomorphism. Since $\phi \mid \mathcal{B}_{0}$ is an irreducible representation of $\mathcal{B}_{0}$, it has the form of conjugation by a unitary $U$ (see [10], p. 38). Thus, conjugation by $U$ and $\phi$ are two ${ }^{*}$-representations of $C^{*}(S)$ that agree on $\mathcal{B}_{0}$. Since $\mathcal{B}_{0} \subseteq C^{*}(S)$ is a non-degenerate ideal, $\phi$ and conjugation by $U$ must agree on $C^{*}(S)$. Hence $S \cong T$.

If $S$ and $T$ are essentially normal, then B-D-F Theory (see [5] or [10]) implies that $S$ and $T$ are unitarily equivalent modulo the compacts if and only if $\sigma_{e}(S)=\sigma_{e}(T)$ and $\operatorname{ind}(S-\lambda)=\operatorname{ind}(T-\lambda)$. Notice, that the condition on the essential spectra is equivalent to requiring that $C^{*}(\pi(S))$ is *-isomorphic to $C^{*}(\pi(T))$ under a map sending $\pi(S) \mapsto \pi(T)$. Thus, the following is a natural question. 
Question 4.8. If $S$ and $T$ are essentially subnormal operators and the map sending $\pi(S) \mapsto \pi(T)$ extends to a *-isomorphism of $C^{*}(\pi(S))$ onto $C^{*}(\pi(T))$ and $S$ and $T$ have the same index function, then are they unitarily equivalent mod the compacts?

The following is also a natural question.

Question 4.9. Can one characterize the essentially subnormal operators that are compact perturbations of subnormal operators? In particular, are they those operators with non-positive index function?

Observe that both questions have positive answers for essential isometries. This follows from Theorem 4.2.

\section{Similarity INVARIANTS}

It was shown in Radjavi and Rosenthal [13] (also see Conway [7], p. 82) that if a subnormal operator is quasisimilar to a normal operator, then it is also normal. We shall use this result to show that within the class of essentially subnormal operators, essential normality is a similiarity invariant. That is, the compactness of the self-commutator is preserved under similarity.

Theorem 5.1. Let $S$ and $T$ be essentially subnormal operators. If $S$ is similar to $T$, then $S$ is essentially normal if and only if $T$ is essentially normal.

Proof. Suppose $T$ is essentially normal and that $S$ and $T$ both act on the same space $\mathcal{H}$. Let $X \in \mathcal{B}(\mathcal{H})$ be an invertible operator such that $X^{-1} S X=T$. Consider $\pi(S), \pi(T)$ and $\pi(X)$ in $\mathcal{B} / \mathcal{B}_{0}$. Let $\phi$ be a faithful representation on a separable Hilbert space of $C^{*}(\pi(S), \pi(T), \pi(X))$.

Now, set $S_{1}=\phi(\pi(S)), N=\phi(\pi(T))$ and $Y=\phi(\pi(X))$. We now have that $S_{1}$ is subnormal, $N$ is normal and $Y$ is invertible. Furthermore, we also have that $Y^{-1} S_{1} Y=N$. Thus, $S_{1}$ is a subnormal operator similar to the normal operator $N$. Hence, $S_{1}$ is also normal (see [13] or Conway [7], p. 82). So $S$ is essentially normal.

One may also extend Theorem 5.1 to essentially hyponormal operators, with a similar proof. This uses a result of Stampfli and Wadha [14] implying that if a hyponormal operator is similar to a normal operator, then it is also normal.

In view of the results for subnormal and hyponormal operators, the following question is natural.

Question 5.2. If $S$ and $T$ are quasisimilar essentially subnormal operators and $S$ is essentially normal, then must $T$ also be essentially normal?

\section{REFERENCES}

[1] A. Aleman, Subnormal Operators with Compact Self-commutator, Manuscripta Math. 91, No. 3 (1996), 353-367. MR 97j:47036

[2] C. Apostol, H. Bercovici, C. Foias and C. Pearcy, Invariant subspaces, dilation theory and the structure of the predual of a dual algebra, J. Funct. Anal. 63 (1985), 369-404. MR 87i:470049

[3] S. Axler and J. Shapiro, Putnam's Theorem, Alexander's spectral area estimate, and VMO, Math. Ann. 271 (1985), 161-183. MR 87b:30053

[4] J. Bram, Subnormal Operators, Duke Math. J. 22 (1955), 75-94. MR 16:835a

[5] L.G. Brown, R.G. Douglas and P.A. Fillmore, Unitary equivalence modulo the compact operators and extensions of $C^{*}$-algebras, Lect. Notes in Math. 345, Springer-Verlag, (1973), 58-128. MR 52:1378 
[6] J. W. Bunce and J.A. Deddens, On the normal spectrum of a subnormal operator, Proc. Amer. Math. Soc. 63 (1977), 107-110. MR 55:8863

[7] J.B. Conway, The Theory of Subnormal Operators, Amer. Math. Soc., Providence, RI, 1991. MR 92h: 47026

[8] J.B. Conway and N.S. Feldman, The Essential Selfcommutator of a Subnormal Operator, Proc. Amer. Math. Soc. 125, No. 1 (1997), 243-244. MR 97c:47022

[9] J.B. Conway and C.R. Putnam, An irreducible subnormal operator with infinite multiplicities, J. Operator Theory 13 (1985), 291-297. MR 86b:47040

[10] K.R. Davidson, $C^{*}$-algebras by Example, Amer. Math. Soc., Providence, RI, 1996. MR 97i: 46095

[11] R. Lange, Essentially Subnormal Operators and K-Spectral Sets, Proc. Amer. Math. Soc. 88 (1983), 449-453. MR 84e:47008

[12] H. Hedenmalm, S. Richter, and K. Seip, Interpolating sequences and invariant subspaces of given index in the Bergman spaces, J. Reine Angew. Math. 477 (1996), 13-30. MR 97i:46044

[13] H. Radjavi and P. Rosenthal, On roots of normal operators, J. Math. Anal. Appl. 34 (1971), 653-664. MR 43:3829

[14] J.G. Stampfli and B.L. Wadhwa, An asymmetric Putnam-Fuglede theorem for dominant operators, Indiana Univ. Math. J. 25 (1976), 359-365. MR 53:14197

[15] L.R. Williams, Restrictions of Essentially Normal Operators, Rocky Mtn J. of Math. 20 No. 2 (1990), 613-618. MR 91g:47015

Department of Mathematics, University of Tennessee, Knoxville, Tennessee 37996 1300

E-mail address: feldman@math.utk.edu

Current address: Department of Mathematics, Michigan State University, East Lansing, Michigan 48824-1027

E-mail address: feldman@math.msu.edu 